\title{
Variación de la calidad del agua y morbilidad durante el proceso de captura y post captura de dos especies de loricáridos comercializados en Acacias (Meta) Colombia Variations in water quality and morbidity during capture and post-capture of loricariids species being sold in Acacias (Meta) Colombia
}

\author{
Sandra L. Parada-Guevara ${ }^{1^{*}}$, Pablo E. Cruz-Casallas ${ }^{2^{*}}$ \\ ${ }^{1}$ Bióloga, MSc, ${ }^{2} \mathrm{MVZ}, \mathrm{MSc}, \mathrm{PhD}$. \\ Instituto de Acuicultura, Grupo de Investigación sobre Reproducción y Toxicología de Organismos \\ Acuáticos, Gritox, Universidad de los Llanos, Villavicencio, Colombia. \\ *Email: salipa11@hotmail.com
}

Recibido: Noviembre 03 de 2010. Aceptado: Agosto 02 de 2011

\section{RESUMEN}

Se realizó un diagnóstico de la actividad pesquera artesanal en el municipio de Acacias-Meta, con énfasis en la captura de hipóstomo (Hipostomus plecostomus) y cucha piña (Panaque maccus), dos especies de loricáridos de la Orinoquia Colombiana que desde hace más de 40 años han sido capturados de manera artesanal y comercializados como peces ornamentales. En este proceso, los individuos capturados presentan tasas de morbilidad y mortalidad que no son registradas, debido a la informalidad de la actividad pesquera, por lo que se pretende determinar los efectos de los procesos de captura y post captura sobre la sobrevivencia de los peces y relacionándola con la calidad del agua en el río y en cada una de las fases de la faena de pesca, abarcando un ciclo hidrológico completo (Febrero 2008-Enero 2009) en los ríos Acacias, Guamal y Orotoy. Desde la captura y hasta las $96 \mathrm{~h}$ post captura, se monitoreó temperatura, $\mathrm{pH}$, oxígeno disuelto, conductividad, amonio, nitritos y la dureza del agua. Se observó que las variables con mayores coeficientes de variación fueron la concentración de amonio $(2.9 \pm 3.2 \mathrm{mg} / \mathrm{l})$, de oxígeno disuelto $(3.2 \pm 2.3 \mathrm{mg} / \mathrm{l})$ y la conductividad $(74.9 \pm 41.5 \mu \mathrm{s})$, las cuales incidieron de manera significativa $(P<0.01)$ sobre la sobrevivencia y calidad de los peces capturados. Adicionalmente, la falta de aclimatación y el manejo inapropiado durante el acopio y el transporte, se identificaron como los factores más críticos asociados con los altos índices de morbilidad ( $10 \%$ y mortalidad (9.5\%). No fueron significativos ( $P>0.05)$ los cambios de condición corporal y de coloración durante las $96 \mathrm{~h}$ de muestreo. Los resultados permiten recomendar la necesidad de aplicar protocolos de manejo orientados a mejorar los procedimientos y condiciones del proceso de captura, que permitan la adaptación de los peces y mejorar su sobrevivencia en cautiverio.

Palabras claves: Pesca, manejo, loricáridos, Colombia, calidad del agua. 


\section{ABSTRACT}

An assessment was made of traditional fishing activity in the town of Acacias in the Meta department, emphasising capturing the suckermouth catfish or common pleco (Hipostomus plecostomus) and Clown pleco/Ringlet pleco/L104/L162/LDA22 (Panaque maccus), two species of the loricariids from the Colombian Orinoco region. They have been traditionally captured and sold as ornamental fish for over 40 years, meaning that captured individuals' morbidity and mortality rates are not registered due to the informality of such fishing. The present work was thus intended to determine the effects of capture and post-capture on the fishes' health, assessing river water quality and every phase of the fishing traditionally used in the area, including a complete hydrological cycle (February 2008-January 2009) in the Acacias, Orotoy and Guamal rivers. Temperature, $\mathrm{pH}$, dissolved oxygen, conductivity, ammonia, nitrites and water hardness were monitored from capture until $96 \mathrm{~h}$ post-capture. It was noted that the variables having the highest coefficients of variation were ammonium ( $2.9 \pm 3.2 \mathrm{mg} \mathrm{L}-1)$, dissolved oxygen (3.2 $\pm 2.3 \mathrm{mg} \mathrm{L}-1)$ and conductivity $(74.9 \pm 41.5 \mathrm{~ms})$, significantly $(p<0.01)$ affecting the survival and health of fish which were caught. Lack of acclimatising the fish and their improper handling during collection and transport were identified as being the most critical factors associated with high morbidity $(10 \%)$ and mortality $(9.5 \%)$. There were significant $(p>0.05)$ changes in body condition and colouring during the $96 \mathrm{~h}$ sampling period. The results lead to recommending the need for management protocols aimed at improving fish capture procedures and conditions to ensure the fishes' adaptation and improve their survival in captivity.

Key words: Fishery, management, loricariid/catfish, Colombia, water quality.

\section{INTRODUCCIÓN}

El municipio de Acacias se encuentra en la región de piedemonte de la Orinoquia colombiana, se ubica a $28 \mathrm{~km}$ al sur de la ciudad de Villavicencio y al occidente del departamento del Meta, a $877090.136 \mathrm{~N}$ y $1036030.558 \mathrm{E}$. En esta localidad se encuentra una amplia distribución, abundancia y riqueza de recursos ictícos, siendo las familias Loricariidae y Characidae las más abundantes (Bogotá, 2004; Bogotá y Maldonado, 2005), las cuales son usadas para consumo humano y como ornamentales. El aprovechamiento de las especies utilizadas como ornamentales se realiza con fines de exportación (Mancera-Rodríguez y Álvarez-León, 2008), por lo que se requiere que las alteraciones producidas en los individuos por los efectos de la captura, transporte y cautiverio sean las mínimas posibles, con el fin de conservar su valor económico

\section{MATERIALES Y MÉTODOS}

Captura y primer acopio. Durante el ciclo hidrológico completo comprendido entre febrero de 2008 y enero de 2009 en los ríos Acacias, Guamal
(Blanco, 2002) y sus ventajas comparativas por patrones de coloración y adaptaciones propias de las especies; por ende, es importante conocer y registrar las condiciones en ambientes naturales de los loricáridos en el río, monitorear los cambios en la calidad de agua (temperatura, $\mathrm{pH}$, oxígeno disuelto, conductividad y amonio) durante la captura y la postcaptura hasta su comercialización, estimar los cambios de condición corporal y de coloración y medir la morbilidad y mortalidad acumulada hasta las $96 \mathrm{~h}$ del segundo acopio, para conocer los puntos críticos del proceso, con la finalidad de evitar morbimortalidad, pérdida de diversidad y prevenir o minimizar el detrimento de la calidad del producto, el que genera ingresos de subsistencia a la comunidad de pescadores de Acacias y los de toda la región.

y Orotoy, se realizó mensualmente una faena de pesca en el cauce central de cada rio. Para realizar los muestreos, inicialmente se escogió un punto 
y a partir de este lugar se tomaron $30 \mathrm{~m}$ aguas arriba y $30 \mathrm{~m}$ aguas abajo, para obtener una franja de $60 \mathrm{~m}$ de longitud por $20 \mathrm{~m}$ en promedio de ancha, para un área total de $120 \mathrm{~m}^{2}$ aproximadamente. Dentro de esta área, tres pescadores realizaron una faena continua de pesca de 180 minutos y se registraron y analizaron las características de los lugares de pesca, de acuerdo con la metodología propuesta por Roldan (1992). La captura se realizó de manera tradicional (careta y "copo"), la cual consiste en que el pescador equipado con una careta de buceo, avista dentro del agua al pez y, posteriormente, con ayuda del "copo" (malla sin nudo de $0.2 \mathrm{~m}$ de diámetro y ojo de malla de 1/4") lo captura sujetándolo manualmente por el dorso. En periodo de aguas altas o en puntos con pastizales y palizadas sumergidas, los pescadores usaron nasas (arte de pesca rectangular, construida en varilla, de $0.36 \mathrm{~m}$ de ancho y $0.50 \mathrm{~m}$ de alto y mango de $0.40 \mathrm{~m}$, con profundidad de seno de 0.77 $\mathrm{m}$ en tela de toldillo) para la captura de los peces.

Después de la captura, los animales fueron depositados en bolsas plásticas, se contaron, se determinaron características externas tales como color, integridad de aletas y escamas, densidad de peces por bolsa y manejo particular de la especie; además, se cuantificó el volumen de agua en cada bolsa y la duración del proceso.

Para el monitoreo de los parámetros fisicoquímicos se adaptó y modificó la metodología de Ajiaco y Ramírez (2000), estableciéndose 15 momentos de evaluación, desde el sitio de captura hasta la comercialización, así: captura (Capt), primer acopio en el rio (Ac01), inicio del transporte hasta la bodega de almacenamiento (Tte1i), llegada a la bodega de acopio (Tte1f); en la bodega se realizaron 8 muestreos a intervalos de $12 \mathrm{~h}$ (Aco2, Aco3, Aco4, Aco5, Aco6, Aco7, Aco8, Aco9); posteriormente se realizaron evaluaciones al inicio del transporte desde la bodega de acopio hasta el comercializador (Tte2i), al final del segundo transporte (Tte2f) y en el acuario de comercialización (com). En cada uno de estos momentos se determinó la calidad del agua, midiendo la concentración de oxígeno disuelto (oximeter YSI® DO 200), pH, temperatura (Digital pHmeter HI 98128, HANNA®), conductividad (conductivímetro monitor Pinpoint $(\AA)$ ), dureza y las concentraciones de amonio total y nitritos (Test Kit Hach $®$ ); así mismo, se registró la mortalidad y se describieron los principales aspectos de manejo.

Primer y segundo transporte. Durante el primer transporte (desde el rio hasta el centro de acopio) y segundo transporte (desde el centro de acopio hasta la bodega del exportador), fueron valorados el tipo de vehículo, la duración y las condiciones de transporte; así mismo, se determinaron los parámetros fisicoquímicos del agua al inicio y al final de cada transporte, el número de ejemplares por bolsa y el número de peces vivos, enfermos y muertos; además, se registraron los cambios de comportamiento de los peces en la bolsa, particularmente la estabilidad del eje y patrón de nado, los cambios de coloración y la integridad de ojos y aletas.

El segundo acopio, se realizó en la bodega de la Cooperativa de pescadores del Municipio de Acacias. Las condiciones y los recipientes usados fueron los mismos que los pescadores han venido utilizando tradicionalmente. Se recibieron los peces en recipientes construidos con canecas plásticas recicladas y cajas de icopor reutilizadas, manteniéndolos sin aireación, sin filtración biológica y sin termostato. Cada $12 \mathrm{~h}$ y hasta las $96 \mathrm{~h}$ post captura $(12,24,36,48,60,72,84$ y 96 h), se evaluó la calidad del agua y se valoraron los peces registrando su estado físico, coloración (se midió con pantones de color RGB word(A) y la condición corporal (buena, regular o mala), estado de las aletas (sanas o deterioradas) estado de los ojos (claros u opacos), el eje de nado (presente o ausente), el reflejo de huída (presente o ausente), la actitud (excitado, normal o decaído) y la presencia o ausencia de lesiones externas (erosión, tumefacción, enrojecimiento, ulceración, hemorragias, así como su magnitud (ligero, moderado y grave). Cada $24 \mathrm{~h}$, según el grado de deterioro del agua, los recipientes fueron sifoneados para retirar los detritos acumulados en el fondo; los volúmenes de agua retirados fueron remplazados diariamente. Los peces enfermos y muertos fueron reportados y retirados. 
Análisis estadístico. Se empleó un análisis descriptivo de carácter unidimensional con el objetivo de establecer la media aritmética y la desviación típica, para contrastar las diferentes condiciones se utilizó el modelo lineal general (GLM) aplicando análisis multivariado de varianza (MANOVA), con contraste canónico de índole ortogonal. Para las variables de índole cualitativas se utilizó

\section{RESULTADOS}

Lugares de pesca. Los lugares de muestreo en los ríos presentaron diferentes tipos de cobertura vegetal, representada por vegetación secundaria relictual, altamente intervenida, con baja representación florística arbórea (8.6\%), compuesta en su mayoría por guamo (Ingasp.), cucharo (Rappaneasp), yarumo (Cecropia sp.), balso (Ochroma lagopus), laurel (Ocotea sp.), cañafisto (Cassia grandis), en estado latizal (DAP -diámetro antero posterior- entre $1.0 \mathrm{y}$ $9.9 \mathrm{~cm}$ ) y fustal (diámetro entre 10 y $19 \mathrm{~cm}$ ) con alturas entre 10 y $15 \mathrm{~m}$, gramíneas y pastos naturales (35.8\%), pastos mejorados (36.8\%) y cultivos de palma de aceite (18.8\%). El hábitat, donde se encontró la mayor cantidad de peces se caracterizó por agua de color claro (58-75\%), corriente suave (50\%) a rápida ( $41.7 \%)$, fondo en piedra de diámetro aproximado de $0.12 \mathrm{~m}$ y rico en materia orgánica ( 58.4 $\%)$ pero con transparencia del $83.3 \%$ al $91.7 \%$ ), características propias de los ríos Acacias y Orotoy. Contrario fue lo observado en el rio Guamal, en el cual las aguas fueron oscuras, turbias y ricas en sedimentos y materia orgánica en descomposición, en virtud que este afluente recibe las aguas servidas vertidas por los municipios de Castilla la Nueva y Guamal. La mayor biodiversidad y fauna béntica asociada se encontró en el rio Acacias, representada por peces de consumo y ornamentales, cangrejos, camarones y moluscos; en la superficie, la mayor parte de fauna estuvo constituida por macro invertebrados y anfibios.

Los valores promedio y desviaciones estándar de las variables fisicoquímicas del agua evaluadas en los tres ríos se describen en la Tabla 1. Los valores presentan ligeras variaciones dependiendo de la época del año, que determina el ciclo hidrológico; sin embargo, no se observaron diferencias estadísticas distribuciones de frecuencia a fin de hallar los porcentajes asociados con cada modalidad adscrita a la variable evaluada. Se relacionaron los parámetros de calidad del agua con la morbimortalidad y el comportamiento de los peces. Se empleó el paquete estadístico SAS versión 9.0. Un valor de $\mathrm{P}<0.05$ fue usado para revelar diferencias significantes.

significativas entre los ríos $(P>0.05)$ para ninguna de estas variables. Las características fisicoquímicas del agua en los ambientes naturales cambiaron en las diferentes épocas del año, mostrando valores de $\mathrm{pH}$ entre 4.8 y 7.5 , temperaturas entre 22 y $28^{\circ} \mathrm{C}$, dureza de 5 a $28 \mathrm{mg} \mathrm{L}^{-1}$ y oxígeno disuelto entre 1 y $5 \mathrm{mg} / \mathrm{l}$. La conductividad y las concentraciones de amonio y nitritos fueron los parámetros de mayor variabilidad en las fuentes naturales. Las concentraciones de amonio variaron $(P<0.05)$ de acuerdo con la época del año, siendo más altas durante la época lluviosa en los tres ríos evaluados.

Captura. Durante todo el año fueron capturados 1597 ejemplares, correspondiendo a 198 hipostomos y 1399 piñas. El rio con mayor porcentaje de captura fue el Acacias con 1235, seguido del rio Orotoy con 276 ejemplares y finalmente el rio Guamal con 86 ejemplares capturados. La cantidad de ejemplares de la especie piña (Figura 1) capturados en el rio Acacias (1172) fue significativamente mayor $(P<0.05)$ comparada con los ríos Guamal (33) y Orotoy (194). La extracción se hizo por inmersión (95\%) o arrastre (5\%), dependiendo de la especie capturada y del volumen y la turbidez del agua (Figura 2). En el primer acopio se observó que la densidad promedio utilizada por el pescador fue de 20 peces/l y la ocurrencia de cambios bruscos de temperatura del agua al terminar la faena de pesca, debido a recambio del $100 \%$ del agua de la bolsa. Se presentaron diferencias estadísticas significativas $(P<0.01)$ en las variables de calidad de agua entre las muestras de los ríos y las de las bolsas donde se alojaron los peces capturados, al igual que en las concentraciones de amonio, $\mathrm{NO}_{2}$ y dureza, en el agua de las bolsas provenientes del río Guamal $(P<0.01)$ con relación a aquellas del Acacias y Orotoy (Tabla 1). 


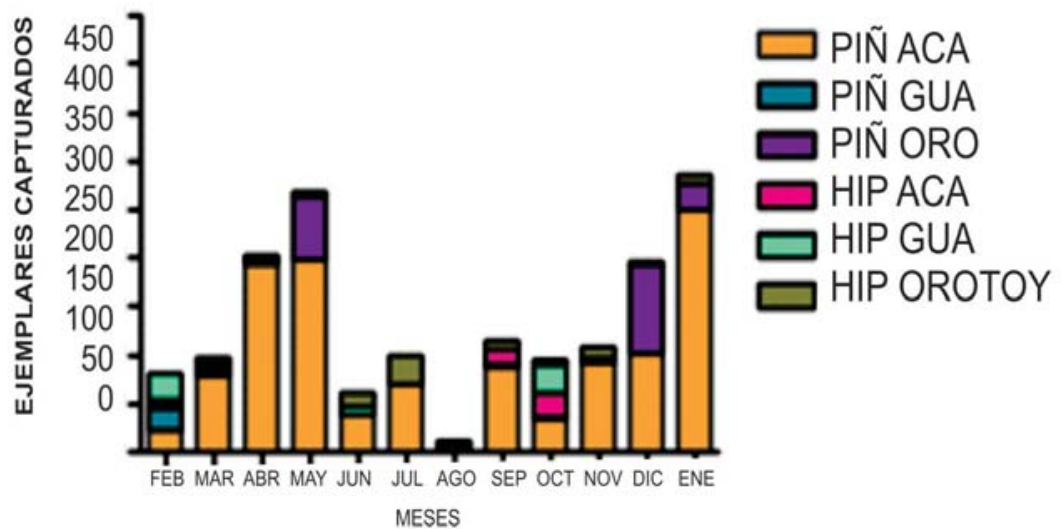

Figura 1. Capturas mensuales de piña e hipóstomo durante el ciclo hidrológico de febrero 2008 a enero 2009, en los ríos Acacias, Guamal y Orotoy en el municipio de Acacias - Meta. Piñ Aca (piñas rio Acacias), Piñ Gua (piñas rio Guamal), Piñ Oro (piñas rio Orotoy), Hip Aca (hipóstomo rio Acacias), Hip Gua (hipóstomo rio Guamal), Hip Orotoy (hipóstomo rio Orotoy)
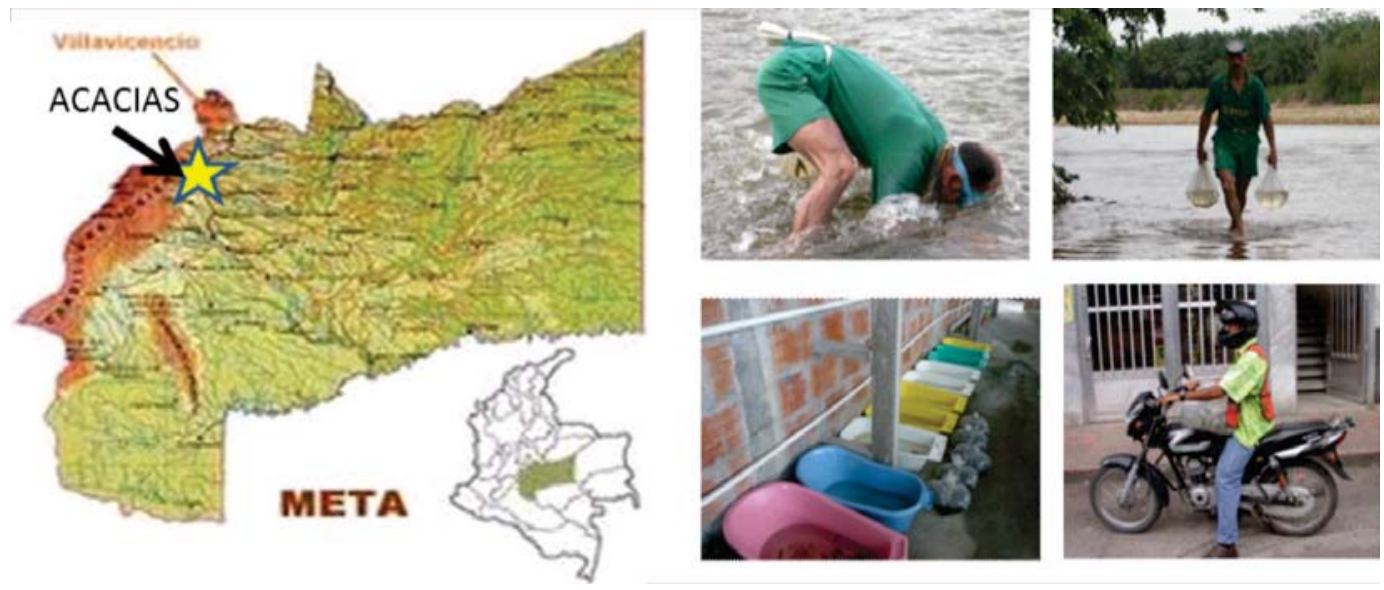

Figura 2. Localización del lugar de pesca $(A)$ y actividades de pesca, transporte y acopio (B)

Tabla 1. Características fisicoquímicas del agua (media \pm DS) tomadas in situ y en las bolsas de captura (BC) en los ríos Acacias, Guamal y Orotoy durante el ciclo hidrológico comprendido entre febrero de 2008 y enero de 2009

\begin{tabular}{lcccccc}
\hline \multicolumn{1}{c}{ Variables } & R. Acacias & R. Guamal & R. Orotoy & BC Acacias & BC Guamal & BC Orotoy \\
\hline OD $\left(\mathrm{mgL}^{-1}\right)$ & $7.2 \pm 0.9$ & $7.5 \pm 1.0$ & $7.7 \pm 1.0$ & $4.0 \pm 1.6$ & $4.4 \pm 1.3$ & $5.8 \pm 5.6$ \\
Temperatura $\left({ }^{\circ} \mathrm{C}\right)$ & $27.1 \pm 1.8$ & $26.1 \pm 2.3$ & $28.4 \pm 2.8$ & $27.0 \pm 3.5$ & $26.9 \pm 1.3$ & $28.2 \pm 2.4$ \\
$\mathrm{pH}$ & $7.1 \pm 0.6$ & $7.0 \pm 0.6$ & $7.0 \pm 0.7$ & $7.2 \pm 3.5$ & $6.8 \pm 0.5$ & $6.9 \pm 0.5$ \\
Conductividad $(\mu \mathrm{s})$ & $19.8 \pm 13.7$ & $29.1 \pm 22.4$ & $39.9 \pm 33.9$ & $26.2 \pm 13.9$ & $22.1 \pm 26.3$ & $36.2 \pm 23.4$ \\
Amonio $\left(\mathrm{mgL}^{-1}\right)$ & $0.2 \pm 0.4$ & $0.3 \pm 0.5$ & $0.2 \pm 0.4$ & $0.3 \pm 0.3$ & $0.5 \pm 0.7$ & $0.27 \pm 0.4$ \\
$\mathrm{NO}_{2}\left(\mathrm{mgL}^{-1}\right)$ & $0.01 \pm 0.01$ & $0.018 \pm 0.6$ & $0.01 \pm 0.01$ & $0.005 \pm 0.007$ & $0.02 \pm 0.04$ & $0.005 \pm 0.008$ \\
Dureza $\left(\mathrm{mgL}^{-1}\right)$ & $19.0 \pm 5.7$ & $19.0 \pm 5.7$ & $22.2 \pm 11.5$ & $18.2 \pm 4.4$ & $18 \pm 3.9$ & $23.4 \pm 11.0$ \\
\hline
\end{tabular}


Transportes. Durante el primer transporte se observó que las bolsas se cerraron sin oxígeno insuflado (100\%) y se embalaron en número de 6 bolsas por cada empaque secundario; en el $100 \%$ de los casos este transporte se realizó en motocicleta, acomodando las bolsas sobre el tanque del vehículo, el cual duró aproximadamente $50 \mathrm{~min}$. Se presentaron diferencias estadísticas significativas $(P<0.01)$ en las variables de calidad de agua, entre el inicio y el final del transporte 1 , siendo las variables más afectadas la concentración de oxígeno disuelto y de amonio, así como la conductividad.

Durante el transporte 2 las condiciones de manejo y calidad del agua variaron debido a los recambios de agua que fueron realizados, al cambio de bolsa, a la adición de oxigeno industrial, a la redistribución de especies por bolsa, a traslados en camiones sin embalaje ni contención y a la larga duración del viaje hasta la bodega del comercializador. La variabilidad mensual se muestra en la Tabla 2.

Tabla 2. Variación de las variables fisicoquímicas (media \pm DS) de calidad de agua durante el procesos de transporte de dos especies de loricáridos capturados en ríos del municipio de Acacias, Meta - Colombia

\begin{tabular}{lcccc}
\hline Variable & Tte1I & Tte1F & Tte2I & Tte2F \\
\hline OD $\left(\mathrm{mgL}^{-1}\right)$ & $4.8 \pm 0.8$ & $2.5 \pm 0.7$ & $4.4 \pm 0.6$ & $7.6 \pm 1.6$ \\
Temperatura $\left({ }^{\circ} \mathrm{C}\right)$ & $28.1 \pm 1.3$ & $27.1 \pm 1.5$ & $23.7 \pm 0,1$ & $26.4 \pm 0.4$ \\
$\mathrm{pH}$ & $6.9 \pm 0.4$ & $6.5 \pm 0.3$ & $8,1 \pm 0.1$ & $7.2 \pm 0.2$ \\
Conductividad $(\mu \mathrm{s})$ & $31.5 \pm 14.2$ & $48.3 \pm 18.1$ & $138.1 \pm 13.4$ & $152.4 \pm 19.5$ \\
Amonio $\left(\mathrm{mgL}^{-1}\right)$ & $0.4 \pm 0.2$ & $1.3 \pm 0.7$ & $0.1 \pm 0.1$ & $1.3 \pm 0.7$ \\
$\mathrm{NO}_{2}\left(\mathrm{mgL}^{-1}\right)$ & $0.01 \pm 0.01$ & $0.01 \pm 0.01$ & $0.02 \pm 0.02$ & $0.02 \pm 0.01$ \\
Dureza $\left(\mathrm{mgL}^{-1}\right)$ & $21.2 \pm 2.4$ & $20.1 \pm 3.4$ & $31.5 \pm 0.6$ & $39.1 \pm 3.1$ \\
\hline
\end{tabular}

Acopio. Al llegar las bolsas a la bodega del segundo acopio, el agua se observó turbia (60\%) y de olor desagradable (100\%). Los animales fueron trasladados a recipientes plásticos reutilizados ( $80 \%$ ) de aproximadamente $30 \mathrm{~L}$ de capacidad, empleando agua de temperatura y $\mathrm{pH}$ diferentes a las de la fuente natural. Después de 3 a 5 días de acopio, los peces nuevamente fueron empacados en bolsas, se les insufló oxígeno y, en algunos casos (20\%), se agregó sal como profiláctico a razón de $2 \mathrm{~g} / \mathrm{l}$. Las variables $\mathrm{pH}$, temperatura, nitritos y dureza no mostraron cambios significativos $(P>0.05)$, mientras que el oxígeno disuelto y la conductividad mostraron cambios importantes durante el acopio $(P<0.01)$ : el amonio fluctuó todo el tiempo entre valores sub-letales y letales. Se presentaron diferencias significativas $(P<0.01)$ en la calidad del agua, dependiendo de la tasa de recambio (100 \% vs. $25 \%$ ); las mayores diferencias se apreciaron cuando el recambio fue de $100 \%$, particularmente en oxígeno disuelto, dureza, conductividad y concentración de amonio
(Tabla 3); las variaciones de calidad de agua, entre el mes de febrero y los meses de julio a noviembre mostraron altas diferencias $(P<0.01)$.

Comercialización. Al llegar al comercializador se observó que el pez nuevamente fue sometido a condiciones de diferente calidad de agua; sin embargo, se mejoró la disponibilidad de oxígeno por la presencia de turbina de aireación (100\%), acondicionamiento del agua por medios químicos, filtros individuales (90\%) para cada recipiente, temperatura controlada por calefactores o extractores $(60 \%)$ y poca manipulación directa del ejemplar.

Manejo general: Los cambios radicales en la calidad del agua ( $100 \%)$, los manejos inapropiados durante el acopio y el transporte, así como la falta de aclimatación (90\%), la mezcla de especies y tamaños (90\%), el almacenamiento en altas densidades (100\%), el uso de recipientes usados (100\%), la ausencia de aireación, calefacción y 
filtros (100\%), las deficiencias en el lavado y desinfección de utensilios (100\%) y la manipulación sin elementos adecuados (90\%), fueron los factores identificados como más críticos y generadores de estrés para el ejemplar y responsables de los índices de morbilidad (10\%) y mortalidad $(9.5 \%)$ observados. Las mortalidades de los peces en el proceso fueron cuantificados, pero por el avanzado estado de descomposición y lisis de los tejidos fueron desechados sin diagnóstico sanitario. Hubo mayor mortalidad en el mes de junio de los hipostomos mientras que en piña se presentaron en diciembre y enero (Figura 3).

Tabla 3. Efecto del porcentaje de recambio de agua sobre sus caracteristicas(media $\pm D S$ ) fisicoquímicas, durante el proceso de acopio de dos especies de loricáridos capturados en ríos del municipio de Acacias, Meta-Colombia

\begin{tabular}{lcccc}
\hline \multicolumn{1}{c}{ Variable } & Recambio 0\% & Recambio 25\% & Recambio 50\% & Recambio 100\% \\
\hline OD $\left(\mathrm{mgL}^{-1}\right)$ & $2.9 \pm 1.9$ & $2.5 \pm 1.6^{\mathrm{a}}$ & $3.5 \pm 2.4$ & $3.7 \pm 3.1^{\mathrm{b}}$ \\
Temperatura $\left({ }^{\circ} \mathrm{C}\right)$ & $24.0 \pm 1.3$ & $23.4 \pm 1.3$ & $23.5 \pm 1$ & $23.8 \pm 1.4$ \\
pH & $7.1 \pm 0.9$ & $7.2 \pm 0.6$ & $6.9 \pm 0.6$ & $7.0 \pm 0.7$ \\
Conductividad $(\mu \mathrm{s})$ & $74.4 \pm 40.7$ & $75.4 \pm 42.8$ & $72.5 \pm 45.1$ & $77.3 \pm 37.7$ \\
Amonio $\left(\mathrm{mgL}^{-1}\right)$ & $2.4 \pm 2.5$ & $2.9 \pm 3.4$ & $2.6 \pm 3.2$ & $3.5 \pm 3.7$ \\
$\mathrm{No}_{2}\left(\mathrm{mgL}^{-1}\right)$ & $0 \pm 0.1$ & $0 \pm 0.1$ & $0.1 \pm 0.1$ & $0.1 \pm 0.1$ \\
Dureza $\left(\mathrm{mgL}^{-1}\right)$ & $30.4 \pm 14.3$ & $34.2 \pm 14^{\mathrm{b}}$ & $30.9 \pm 14.5$ & $25.5 \pm 11.2^{\mathrm{a}}$ \\
\hline
\end{tabular}

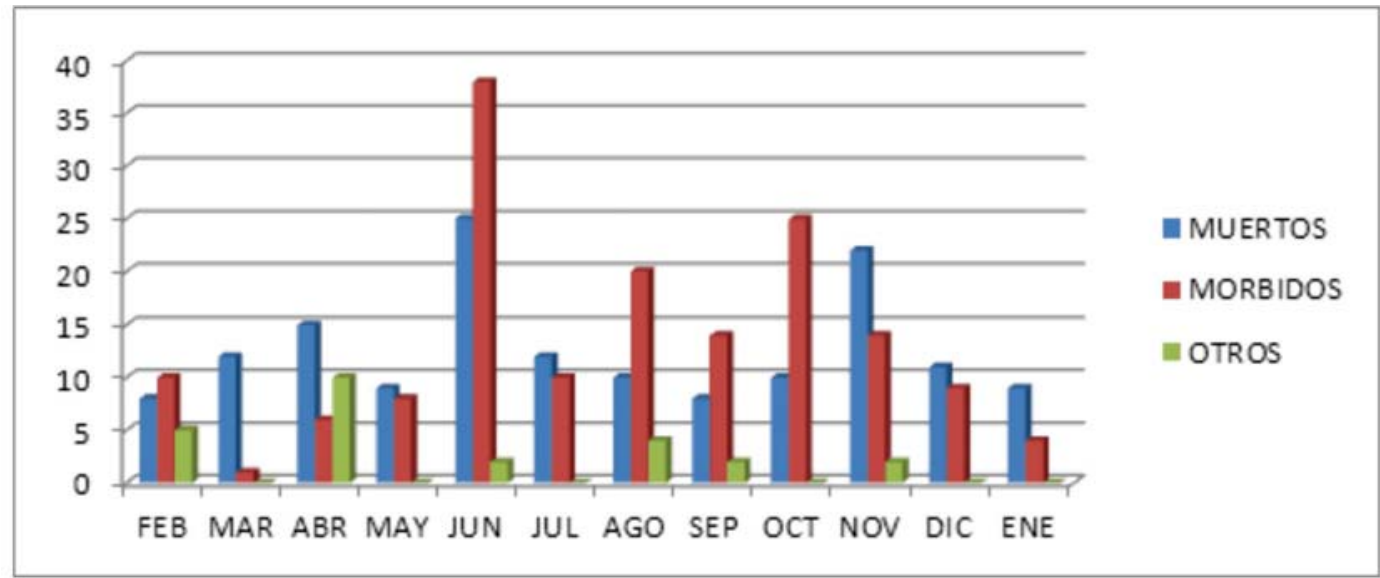

Figura 3. Morbilidad y mortalidad de dos especies de loricáridos, durante el proceso de captura y postcaptura en el municipio de Acacias, Meta - Colombia

Durante el acopio se observó aumento progresivo de la conductividad del agua, pasando de $29.6 \mu \mathrm{s}$ a $155.9 \mu$ s (c.v. 110.84), pero sin efectos aparentes sobre los peces. Las variaciones en las concentraciones de oxígeno disuelto (Figura 4) oscilaron de la normoxia $\left(4.00 \mathrm{mg} \mathrm{L}^{-1}\right)$ a la hipoxia (1.95 $\left.\mathrm{mg} \mathrm{L}^{-1}\right)$ y a la hiperoxia $\left(7.66 \mathrm{mg} \mathrm{L}^{-1}\right)$, afectando la calidad y la sanidad del pez, manifestada por deterioro de las aletas y branquias.
El aumento en la concentración de amonio se apreció al finalizar el primer transporte y el segundo acopio, cuando se inició el transporte 2 y al terminar el segundo acopio (Figura 5). La mayor concentración de amonio se observó cuando las bolsas permanecieron más tiempo empacadas como sucedió durante el transporte hasta Bogotá, en los meses de Junio a Septiembre, cuando se entregaron al exportador. Durante el monitoreo del segundo acopio (Tabla 4), se observó un aumento 
en la concentración de amonio a medida que transcurrió el tiempo. Para contrarrestar el incremento de esas concentraciones, se recurrió a realizar en las primeras $24 \mathrm{~h}$ un recambio del $100 \%$. La concentración de nitritos no mostró variaciones significativas.

Con relación a los cambios de condición corporal y de coloración de los peces durante las primeras
$96 \mathrm{~h}$ post-captura, se observó un ligero cambio en la tonalidad del color, del cuerpo, el cual no fue significativo durante el tiempo de almacenamiento estudiado ( $P>0.05$ ). La condición corporal se valoró mediante la apreciación de la masa corporal y la apariencia robusta del área delimitada por las vértebras, las costillas dorsales y el pedúnculo caudal. No se apreció cambio en la contextura del animal en las $96 \mathrm{~h}$ de duración del monitoreo.

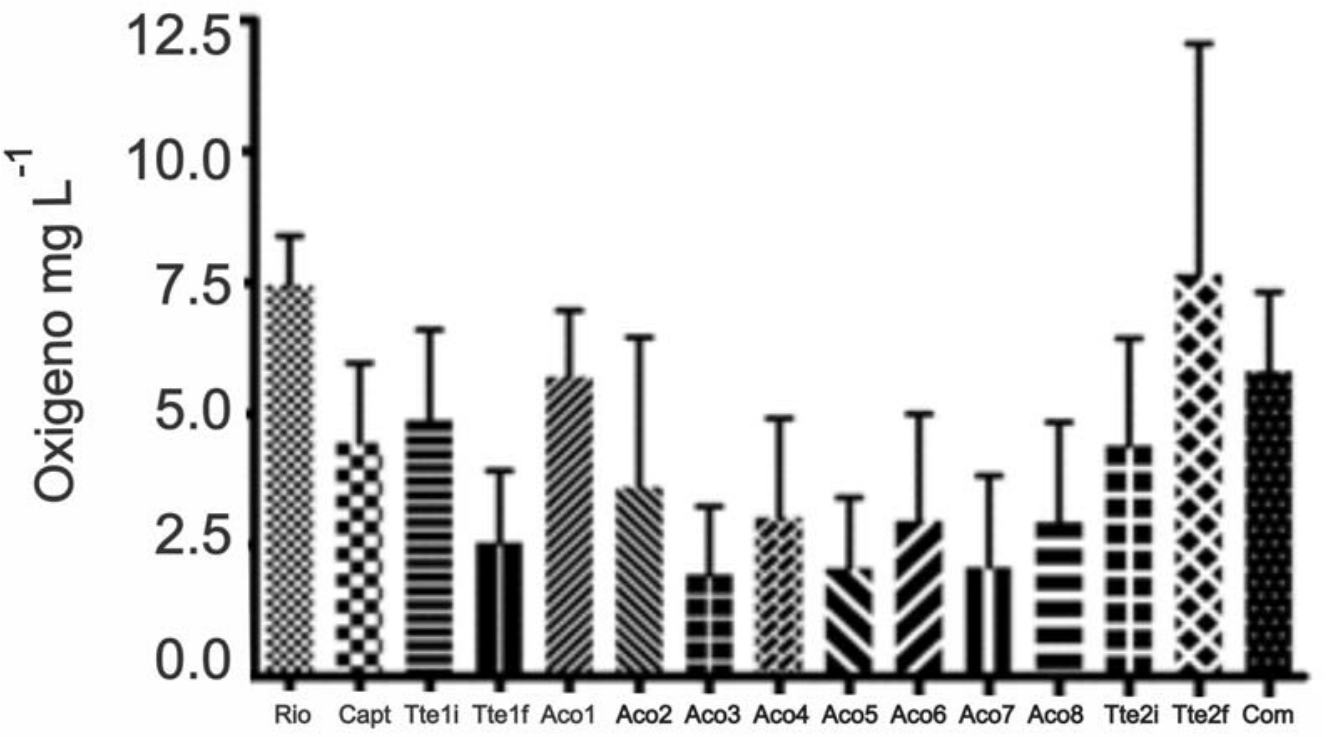

Momentos de captura y post captura

Figura 4. Variación en la concentración de oxígeno disuelto, durante el proceso de captura y postcaptura de dos especies de loricáridos, en el municipio de Acacias, Meta - Colombia

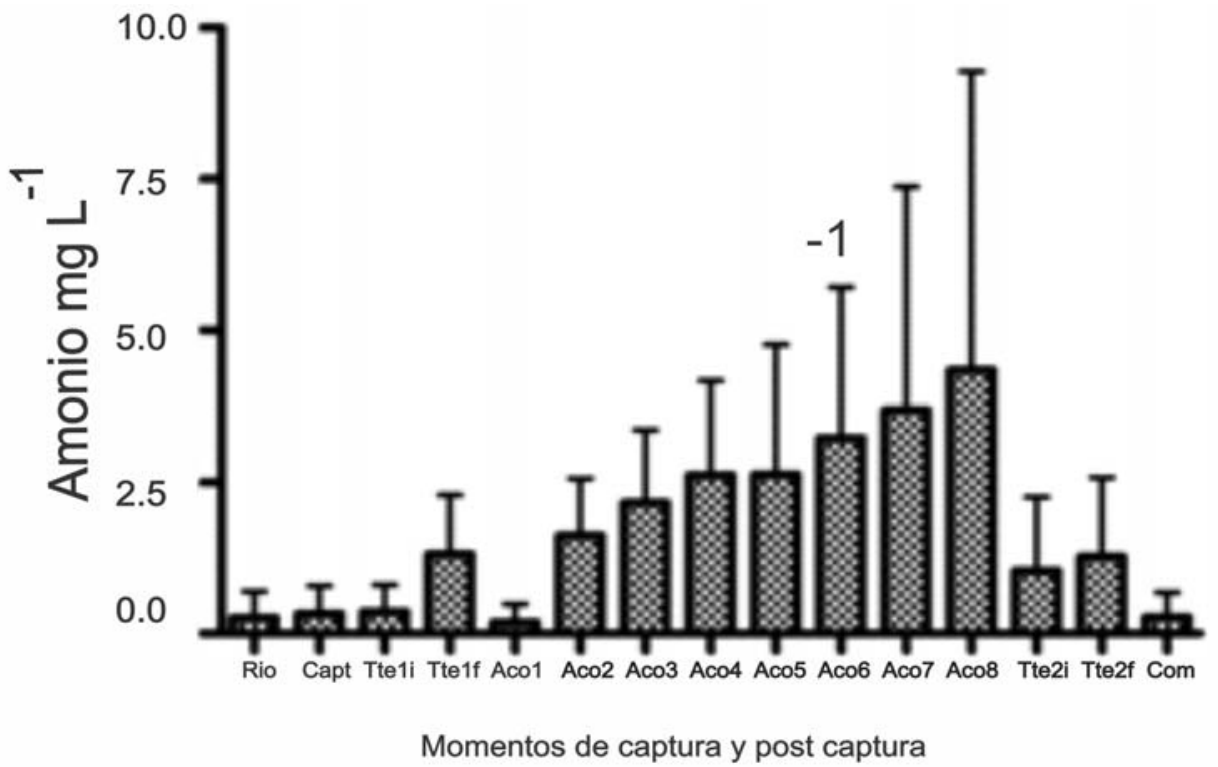

Figura 5. Variación en la concentración de amonio, durante el proceso de captura y postcaptura de dos especies de loricáridos, en el municipio de Acacias, Meta - Colombia 
Tabla 4. Variación de las variables fisicoquímicas (media \pm DS) de calidad de agua durante las actividades de captura y postcaptura de dos especies de loricáridos comercializados en el municipio de Acacias, Meta - Colombia

\begin{tabular}{lccccccc}
\hline & $\begin{array}{c}\mathbf{O D} \\
\left(\mathrm{mgL}^{-1}\right)\end{array}$ & $\mathbf{T}^{\circ}\left({ }^{\circ} \mathrm{C}\right)$ & $\mathbf{p H}$ & Cond $(\boldsymbol{\mu s})$ & $\begin{array}{c}\text { Amonio } \\
\left(\mathrm{mgL}^{-1}\right)\end{array}$ & $\mathbf{N O}_{2}\left(\mathrm{mgL}^{-1}\right)$ & $\begin{array}{c}\text { Dureza } \\
\left(\mathrm{mgL}^{-1}\right)\end{array}$ \\
\hline Rio & $7.5 \pm 0.9$ & $27.2 \pm 2.4$ & $7.0 \pm 0.6$ & $29.6 \pm 25.4$ & $0.3 \pm 0.4$ & $0.06 \pm 0.3$ & $20.2 \pm 8.1$ \\
Captura & $4.5 \pm 1.5$ & $27.5 \pm 2.9$ & $7.1 \pm 2.4$ & $29.1 \pm 21.1$ & $0.3 \pm 0.5$ & $0.01 \pm 0.02$ & $20.1 \pm 7.8$ \\
Tte1I & $4.9 \pm 1.7$ & $28.1 \pm 3.0$ & $7.1 \pm 2.4$ & $31.6 \pm 21.2$ & $0.4 \pm 0.4$ & $0.009 \pm 0.02$ & $21.6 \pm 8.8$ \\
Ttelf & $2.6 \pm 1.4$ & $27.3 \pm 3.3$ & $6.5 \pm 0.6$ & $49.2 \pm 31.5$ & $1.3 \pm 1.9$ & $0.01 \pm 0.02$ & $20.4 \pm 8.0$ \\
Acopio & $5.7 \pm 1.3$ & $25.1 \pm 1.0$ & $7.8 \pm 1.5$ & $63.7 \pm 39.5$ & $0.2 \pm 0.3$ & $0.004 \pm 0.006$ & $27.6 \pm 1.8$ \\
1 (rí) & & & & & & & \\
Acopio & $3.6 \pm 2.9$ & $23.0 \pm 0.7$ & $7.1 \pm 0.6$ & $61.3 \pm 32$ & $1.6 \pm 0.9$ & $0.016 \pm 0.020$ & $29.9 \pm 13.5$ \\
2 Aco1 & & & & & & & \\
Aco2 & $1.9 \pm 1.3$ & $24.6 \pm 1.1$ & $6.9 \pm 0.6$ & $65.0 \pm 29.5$ & $2.2 \pm 1.2$ & $0.05 \pm 0.1$ & $30.5 \pm 12.5$ \\
Aco3 & $3.0 \pm 1.9$ & $22.8 \pm 0.7$ & $7.0 \pm 0.6$ & $68.2 \pm 33.0$ & $2.6 \pm 1.5$ & $0.07 \pm 0.15$ & $29.8 \pm 13.0$ \\
Aco4 & $2.1 \pm 1.3$ & $24.6 \pm 1.0$ & $6.9 \pm 0.7$ & $81.0 \pm 37.9$ & $2.6 \pm 2.1$ & $0.06 \pm 0.12$ & $31.7 \pm 17.2$ \\
Ac05 & $3.0 \pm 2.0$ & $23.0 \pm 0.7$ & $7.0 \pm 0.6$ & $80.5 \pm 41.0$ & $3.2 \pm 2.5$ & $0.07 \pm 0.1$ & $29.8 \pm 14.0$ \\
Aco6 & $2.1 \pm 1.7$ & $24.8 \pm 1.0$ & $7.0 \pm 0.6$ & $85.9 \pm 46.8$ & $3.7 \pm 3.7$ & $0.06 \pm 0.1$ & $29.6 \pm 14.5$ \\
Aco7 & $2.9 \pm 1.9$ & $23.1 \pm 0.8$ & $7.1 \pm 0.6$ & $91.4 \pm 52.0$ & $4.3 \pm 4.9$ & $0.06 \pm 0.09$ & $29.8 \pm 14.7$ \\
Tte2in & $4.4 \pm 2.0$ & $23.8 \pm 0.6$ & $8.1 \pm 1.2$ & $143.4 \pm 159.0$ & $1.0 \pm 1.2$ & $0.02 \pm 0.08$ & $30.8 \pm 17.1$ \\
Tte2fi & $7.7 \pm 4.4$ & $26.5 \pm 1.8$ & $7.2 \pm 0.5$ & $155.9 \pm 180.5$ & $1.3 \pm 1.3$ & $0.01 \pm 0.01$ & $39.6 \pm 15.5$ \\
Com & $5.8 \pm 1.5$ & $26.1 \pm 1.1$ & $7.3 \pm 0.2$ & $102.1 \pm 88.9$ & $0.3 \pm 0.4$ & $0.01 \pm 0.03$ & $41.3 \pm 13.2$ \\
\hline
\end{tabular}

\section{DISCUSIÓN}

La pesca ornamental es una actividad económica de la Orinoquia colombiana, que aporta el $88 \%$ del total nacional (Galvis-Vergara et al, 2007). Se capturan aproximadamente 97 especies pertenecientes a 19 familias, siendo los loricáridos los más representativos con 27 especies. En el año 2009 esta actividad generó ingresos de aproximadamente 8 millones de dólares $(\mathrm{CCl}$, 2009); no obstante, su extracción y manejo se realizan de manera artesanal y el comercio depende de la demanda del mercado internacional y de la oferta ambiental (Blanco, 1992; 2002; Ramírez y Ajiaco, 2001); sin embargo, los ingresos que reciben los pescadores por esta actividad sólo alcanzan el nivel de subsistencia (Parada, 2009). Por otra parte, no se llevan estadísticas confiables de este comercio y no existe el conocimiento biológico y ecológico suficiente, que permita su aprovechamiento sostenible (Mancera-Rodríguez y Álvarez-León, 2008).

Los estudios en ambientes naturales para la región de piedemonte son escasos; Sanabria y et al. (2007) con base en estadísticas de 2004 reportaron condiciones semejantes a las encontradas en los ríos Guamal y Orotoy en este estudio: temperatura entre $21.8^{\circ} \mathrm{C}$ y $30^{\circ} \mathrm{C}$, conductividad entre 16 y $34.1 \mu \mathrm{s}, \mathrm{pH}$ entre 5.5 y 6,0 y sólidos disueltos entre 7 y 16 ppm. Por su parte, las características bioecológicas de los lugares de captura fueron semejantes a las reportados por Blanco (1992), Ajiaco y Ramírez (2000), Ramírez et al. (2001), Parada y Murillo (2005), Sanabria (2007), Ramírez y Ajiaco (2008) y Parada (2008). 
Las labores de captura y post captura, son semejantes a las reportadas para la altillanura (Blanco, 1992; Ajiaco y Ramírez, 2000; Ramírez et al. 2001; Parada y Murillo, 2005; Sanabria, 2005; 2007; Ramírez y Ajiaco, 2008), siendo que las pocas diferencias radican en la especialización de la pesca con careta y copo, la limitación a pocas especies de loricáridos, callíctidos, gymnótidos y potamotrygónidos, el medio de transporte usado (motocicleta), los recipientes de acopio (canecas plásticas reusadas) y la ausencia de profilácticos y de medicamentos durante el proceso (Parada, 2008).

Durante la evaluación de los procesos de captura y postcaptura se evidenció que el amonio, el oxígeno disuelto y la conductividad, junto con las deficientes condiciones de aseo y manejo, ejercen una fuerte influencia sobre la sanidad y calidad de los peces comercializados. La temperatura y el $\mathrm{pH}$ mostraron constantes variaciones durante los 15 momentos estudiados; sin embargo, se mantuvieron dentro de los rangos permisibles $\left(\mathrm{T}^{\circ}: 24-26^{\circ} \mathrm{C}, \mathrm{pH} 6\right.$ - 8 ) para la adaptación y el manejo de peces tropicales (Wedler, 1998; Ajiaco y Ramírez, 2000); no obstante Vinatea (2002) sugiere que el aumento de un grado en la temperatura o de una unidad en el valor de $\mathrm{pH}$ puede aumentar la toxicidad del $\mathrm{NH}_{3}$ entre un 5 y un $10 \%$, lo que puede reflejar un posible aumento en la morbilidad y en el deterioro de los peces acopiados. La saturación de oxígeno, así como la hipo e hiperoxigenación observadas durante el transporte y acopio en este estudio, puede llevar a modificaciones en la toxicidad del amoniaco (Tomasso, 1994; Wedemeyer, 1997) y ocasionar estrés e inmunodepresión, haciendo al pez más susceptible a infecciones y parasitosis (Barton, 1997).

Según Randall (2002) y Lim (2003) una concentración superior a $0.20 \mathrm{mg} / \mathrm{l} \mathrm{de} \mathrm{NH}_{3}$ ocasiona trastornos respiratorios en una exposición aguda ocasionando perturbaciones de comportamiento, inquietud, aumento de la agresividad, desorientación, espasmos y crisis convulsivas, posiblemente por sus efectos neurológicos, lo cual puede explicar en los loricáridos la dificultad respiratoria (boqueo), los espasmos, la pérdida de eje nado y el deterioro del pez. La $\mathrm{CL}_{50}$ del amoniaco no ionizado $\left(\mathrm{NH}_{3}\right)$ reportado para ciprínidos y salmónidos varía entre 0.083 y $4.6 \mathrm{mg} / \mathrm{l}$ (Vinatea, 2002), dependiendo de factores como la especie, el $\mathrm{pH}$ y la temperatura del agua, la saturación de $\mathrm{O}_{2}$ y $\mathrm{CO}_{2}$, la salinidad (Ladon, 1998) y el grado de adaptación del animal. Igualmente, una exposición prolongada al amoniaco puede causar la muerte de los ejemplares; por lo tanto, es importante determinar el comportamiento de estas variables en estas especies para diseñar futuras estrategias de manejo.

Los porcentajes de mortalidad durante todo el proceso de acopio y transporte encontrados en Acacias fueron altos, comparados con los encontrados en Villavicencio del $0 \%$ (Blanco, 1992), 1,5\% en Puerto Gaitán (Ajiaco y Ramírez, 2000), 4,9 \% en Inírida (Ramírez, 2000, 2001) y 2 al $5 \%$ en Puerto Carreño (Ramírez et al. 2001;2002). La mortalidad reportada por Ramírez (2000) en Puerto Inírida fue atribuida a los cambios de temperatura y a las bajas concentraciones de oxígeno disuelto. Posiblemente esté sucediendo lo mismo en el municipio de Acacias (9.5\%), a lo cual hay que sumar las pobres condiciones sanitarias (Parada, 2008). La mayor ocurrencia de morbilidad y mortalidad de loricáridos se observó durante el primer transporte y el segundo acopio, situación posiblemente originada por los cambios bruscos en la calidad del agua en la bolsa de transporte y en el recipiente de acopio; a la alta densidad de alojamiento de los peces, a la frecuente manipulación, el acúmulo de tóxicos metabólicos y a la presencia de patógenos oportunistas (Ajiaco y Ramírez, 2000).

La luminosidad no fue una variable medida, pero se evidenció que puede influir sobre el comportamiento de los peces, debido a que tienden a aglomerarse, en busca de aéreas de sombra o refugio, aumentando la competencia por espacio y oxígeno, así como lo reportó Ramírez-Duarte et al. (2009). La ausencia de protocolos de manejo, las malas prácticas de lavado y desinfección de instalaciones, bolsas, recipientes y utensilios, el desconocimiento de la biología y del comportamiento de las especies, el desconocimiento de la sintomatología y de los patógenos, también contribuyen a disminuir la calidad de los peces y por lo tanto afectan la comercialización de los mismos (Parada, 2009a). 


\section{CONCLUSIÓN}

El agua del rio Orotoy presenta mayor temperatura, conductividad y dureza, que los otros dos ríos estudiados, debido posiblemente a los efectos de los vertimientos de las aguas asociadas a la producción de hidrocarburos de los campos petroleros 1 y 2 del Campo Castilla, operados por la compañía Ecopetrol. El río Guamal está altamente impactado por las descargas de aguas servidas de los municipios circundantes. Sin embargo, las diferencias en las condiciones fisicoquímicas del agua de los ríos no inciden significativamente en la sanidad y calidad de los peces extraídos, pero si en su diversidad, riquezay abundancia.

\section{AGRADECIMIENTOS}

A la Cooperativa Coopesca, a la Universidad Nacional de Colombia, a Manos unidas ONGD y a la Corporación Kotsala, que con los proyectos de investigación "Construcción del primer mapa

\section{REFERENCIAS}

Ajiaco RE, Ramírez H. 2000. Detección de factores causantes de morbilidad y mortalidad de peces ornamentales en la captura, acopio y transporte en Puerto Gaitán - Villavicencio, Meta. Informe. Centro de investigación y fomento de la pesca artesanal y la acuicultura-CFPA. 45 p.

Barton, BA. 1997. Stress in finfish: past, present and future- a historical perspective. p. 1-33. In: Iwama, GK, Pickering AD, Sumpter JP, Schreck CB. (Ed). Fish stress and health in aquaculture. Society for Experimental Biology. Cambridge University Press. U.K. 73 p.

Blanco MC. 1992. Ordenamiento de las pesquerías de los peces ornamentales en los llanos orientales. Informe técnico. Instituto Nacional de Recursos Naturales Renovables- INDERENA. Bogotá. 200 p.

Blanco MC. 2002. Consideraciones sobre los peces ornamentales de Colombia. Libro rojo de peces dulceacuícolas de Colombia. Mojica JJ, Castellanos C, Usma JS (ed): 47-54.

Bogotá JD. 2004. Contribución al conocimiento de la ictíofauna de la Amazonia y la Orinoquia colombiana:
El manejo del pez durante la captura, transporte y acopio tiene una alta incidencia sobre la calidad del ejemplar. Este seguimiento y valoración de las variaciones de calidad del agua durante el proceso de captura y postcaptura permitieron conocer los puntos críticos y las posibles causas, lo cual facilitará iniciar un proceso de implementación de protocolos de pesca, transporte y acopio, que deben ser adoptados por todos los pescadores, con el fin de mejorar la calidad del producto, garantizar la sostenibilidad del recurso, aumentar los ingresos de la actividad pesquera y permitir un desarrollo social del gremio.

epidemiológico de las enfermedades de los peces ornamentales en Colombia" MADR-057-2007U4448387-07 y "Mejoramiento del sistema de pesca artesanal en Acacias. Meta" COL 602 XLIXB (116), hicieron posible el desarrollo de este trabajo.

colección ictíca del instituto de Investigaciones de recursos biológicos Alexander von Humboldt. Trabajo de Grado. Universidad de Bogotá Jorge Tadeo Lozano.

Bogotá JD, Maldonado-Ocampo JA. La colección de peces del instituto Alexander von Humboldt (IAVH), nuevos registros y representatividad. Parte I: Orinoquia. En: Dahlia-Rev Asoc Colomb Ictiol. 2005; 8: 29-37.

Corporación Colombia Internacional- CCl. 2009. Pesca yAcuicultura de Colombia. 2009. Corporación Colombia Internacional. Ministerio de Agricultura. 125p.

Galvis-Vergara G, Mojica-Corso JI, Provenzano-Rizzi F, Lazzo-Alcala C, Taphorn D, Royero R, CastellanosCastillo C, et al. 2007. Peces de la Orinoquia colombiana con énfasis en especies de interés ornamental. (Editores generales: Sanabria-Ochoa AL, Victoria-Daza P, Beltrán IC). Instituto Colombiano de Desarrollo Rural INCODER y universidad Nacional de Colombia. Primera edición.

Ladon S. 1998. Transport of fish in bags. Aquaculture Extension. Illinois -Indiana. Asea grant program. Fact sheet as-462-w. 
Lim LC, Dhert Py Sorgeloos P. Recent developments and improvements in ornamental fish packaging systems for air transport. Aquaculture Research. 2003;34: 923-935.

Mancera-Rodríguez NJ. y Álvarez-León R. Comercio de peces ornamentales en Colombia. Acta Biol Colomb. 2008;13:23-52.

Parada SL y Murillo Jl. 2005. Informe final proyecto: Establecer medidas de manejo y aprovechamiento racional para el recurso pesquero de tipo ornamental in-situ y desarrollar sistemas de manejo ex-situ, con el fin de garantizar la sostenibilidad del recurso en la comunidad de Puerto Caldas. Agosto. Contrato 2.7.04115. Informe institucional Cormacarena. $350 \mathrm{p}$.

Parada SL. 2008. Informe final. Proyecto: Seguimiento y monitoreo de prácticas de acopio de peces ornamentales en Acacias, Meta. Instituto HumboldtSena. Mayo 2008-agosto 2008. 64 p.

Parada SL. 2009. Informe final. Proyecto: Mejoramiento del sistema de pesca artesanal en Acacias. Meta. Corporación Kotsala. Manos unidas ONGD (España). 1 de Agosto de 2008-31 Julio de 2009. 80 p.

Parada SL. 2009a. Manual para cinco especies capturadas en Acacias. Manejo biológico, sanitario y alimenticio. Corporación Kotsala. Manos unidas ONGD (España). $27 \mathrm{p}$.

Ramírez-D WF, Parada SL, Martínez N, Eslava-M PR, Figueroa J, Iregui C. 2009. Manejo de peces de la familia Loricaridae en el Municipio de Acacias, Meta: monitoreo desde la captura hasta la comercialización (Reporte preliminar). Memorias XV jornada de Acuicultura. Universidad de los Llanos. Octubre 8 y 9.

Ramírez-Gil H. 2000. Evaluación de la actividad pesquera ornamental en el área de influencia de Inírida, orientado al desarrollo ordenado de este sector económico. Instituto Nacional de Pesca y Acuicultura - INPA. Contrato INPA-PRONATTAn임 944104. 14 p. www.pronatta.gov.co. Búsqueda realizada el 27 de Octubre de 2009.

Ramírez-Gil Hy Ajiaco RE. (ed). 2001. La pesca en la baja Orinoquía colombiana: una visión integral. INPA. $255 p$.
Ramírez-Gil Hy Ajiaco RE. La pesca en la Orinoquía colombiana: pasado, presente y futuro. Boletín científico. 2002;7:239-270. INPA.

Ramírez-Gil Hy Ajiaco RE. 2008. Aspectos biológicos y de manejo de los peces ornamentales. Contribución a la gestión sostenible y al conocimiento biológico y socio económico de la cadena de valor de peces ornamentales de Puerto Carreño, Reserva de biosfera El Tuparro (Vichada-Colombia) Fundación OmachaFundación Horizonte Verde. Bogotá. Colombia. 125p.

Randall DJ y Tsui TKN. Ammonia toxicity in fish. Marine Pollution Bulletin 2002;45:17-23.

Roldan G. 1992. Fundamentos de Limnología Neotropical. Universidad de Antioquia. Medellín: Universidad de Antioquia, $109 p$.

SanabriaAl. 2005. Aprovechamiento del recurso íctico ornamental en Colombia. En Memorias: Taller Internacional de Peces Ornamentales. Bogotá. Agosto 24-26.

SanabriaAl, Daza PV y Beltrán IC. (eds.). 2007. Peces de la Orinoquia Colombiana con énfasis en especies de interés ornamental. INCODER. Universidad Nacional de Colombia. Bogotá, 425 p.

Tomasso JR. Toxicity of nitrogenous wastes to aquaculture animals. Reviews in Fisheries Sciences. 1994;2:291-314.

Vinatea L. 2002.Principios químicos de calidad del agua en Acuicultura. Una revisión para peces y camarones. CBS. Manual 13. Universidad autónoma Metropolitana. Div. Ciencias Biológicas y de la salud. México. 94 p.

Wedemeyer $\mathrm{G}$. Effects of rearing conditions on the health and physiological quality of fish in intensive culture. In: fish stress and health in aquaculture (ed. By Iwama GK, Pickering AD, Sumpter JP y Schreck CB): 35-71. Cambridge university press. En: Lim LC, Dhert P y Sorgeloos P. 2003. Recent developments and improvements in ornamental fish packaging systems for air transport. Aquaculture Research. 1997;34: 923-935.

Wedler E. 1998. Introducción en la acuicultura con énfasis en los neotrópicos. Corpamag. Santa Marta. $388 \mathrm{p}$. 